\title{
Electrochemically Assisted Maskless Selective Removal of Metal Layers for Three-Dimensional Micromachined SOI RF MEMS Transmission Lines and Devices
}

\author{
Mikael Sterner, Niclas Roxhed, Member, IEEE, Göran Stemme, Fellow, IEEE, and \\ Joachim Oberhammer, Member, IEEE
}

\begin{abstract}
This paper presents a novel electrochemically assisted wet-etching method for maskless selective removal of metal layers. This method has been developed as the key process step for enabling the fabrication of low-loss 3-D micromachined silicon-oninsulator-based radio-frequency microelectromechanical systems transmission line components, consisting of a silicon core in the device layer covered by a gold metallization layer. For this application, the full-wafer sputtered metallization layer must be locally removed on the handle layer to guarantee for a well-defined and low-loss coplanar-waveguide propagation mode in the slots of the transmission line. It is not possible to use conventional photolithography or shadow masking. Gold areas to be etched are biased by a 1.2-V potential difference to a saturated calomel reference electrode in a $\mathrm{NaCl}(\mathrm{aq})$ solution. The measured etch rate of the proposed local electrochemically biased etching process is $520 \mathrm{~nm} / \mathrm{min}$, and no detectable etching was observed on unbiased areas even after a 1-h etch. The suitability of different adhesion layers has been investigated, and Ti-based adhesion layers were found to result in the highest yield. The new etching method has been successfully applied for the fabrication of transmission lines with integrated microswitches, lowering the insertion loss of the waveguide at $10 \mathrm{GHz}$ from 1.3 to $0.3 \mathrm{~dB} / \mathrm{mm}$. The issue of unwanted thin metallic connections caused by secondary deposition during sputtering is discussed but found not to significantly affect the process yield. Finally, local removal of gold on isolated features even within the device layer is presented for locally removing the metallization on stoppers of laterally moving electrostatic actuators, to drastically reduce the mechanical wear on stopper tips.

[2010-0326]
\end{abstract}

Index Terms-Coplanar waveguide (CPW), radio-frequency (RF) microelectromechanical systems (MEMS), transmission lines.

\section{INTRODUCTION}

$\mathbf{T}$ RANSMISSION lines are used to propagate radiofrequency (RF) signals. Coplanar waveguide (CPW) transmission lines, consisting of a signal line between two ground conductors arranged in the same plane, allow for easy integration on any dielectric substrate and low-loss transmission for a large bandwidth up to $100 \mathrm{GHz}$ [2]. Three-

Manuscript received November 23, 2010; revised March 31, 2011; accepted May 11, 2011. Date of publication July 7, 2011; date of current version August 3, 2011. Subject Editor F. Ayazi.

The authors are with the Microsystem Technology Laboratory, Royal Institute of Technology (KTH), 10044 Stockholm, Sweden (e-mail: msterner@kth.se).

Color versions of one or more of the figures in this paper are available online at http://ieeexplore.ieee.org.

Digital Object Identifier 10.1109/JMEMS.2011.2159100 dimensional micromachining allows for the fabrication of transmission lines which are characterized by extremely low losses [3]. The substrate losses are reduced either by suspending the signal line above the substrate or by etching the substrate below the transmission line. Furthermore, ohmic losses are reduced by having thick high-aspect-ratio structures with tall sidewalls, allowing a larger volume for the crowded signal current, which is laterally confined by the skin effect to a small volume on the edge of conventional thin signal lines. The diverse fabrication techniques employed for 3-D transmission lines include both surface micromachining and bulk micromachining, ranging from molded polymer core electroplated structures [4] to $\mathrm{KOH}$ etched grooves in Si substrates [5].

Micromachined transmission lines in silicon-on-insulator (SOI) have been demonstrated for switches [6], and the authors of this paper have demonstrated coplanar-transmission-lineembedded mechanically multi-stable single-pole-double-throw switch mechanisms [7], [8], as shown in Fig. 1.

Electrochemical processes are heavily used in microfabrication because of their cost effectiveness, uncomplicated setups, and high selectivity [9]. Additive (cathodic) reactions are used for electrodeposition of metal layers [10], through-mask electroplating for mold replication [11], and electroforming for creating 3-D shapes [12]. Subtractive (anodic) reactions are used for electropolishing to level out surfaces [13], electrochemical micromachining in the proximity of a precision shaped tool [14], and electrochemical etching through a resist mask [15].

In this paper, we make use of electrochemically discriminated dissolution of gold in a chloride solution. Above a certain electrode potential, gold is dissolved as a $\mathrm{Au}(\mathrm{III})$ complex [16], [17]

$$
\mathrm{Au}(\mathrm{s})+4 \mathrm{Cl}^{-}(\mathrm{aq}) \rightarrow \mathrm{AuCl}_{4}^{-}(\mathrm{aq})+3 e^{-} .
$$

This reaction has been used in microfabrication for electrochemical etching of seed layers [18] and for localized release of $\mathrm{Au}-\mathrm{Si}$ eutectically bonded microstructures [19]. It has also been proposed for in vivo release of drugs [20]. In this paper, we propose the use of maskless electrochemically assisted wet etching for localized removal of unwanted metal areas.

The basic idea of this paper was first introduced by the authors at a technical conference [1]. This paper has been enhanced with a detailed discussion on the effect of residual gold 


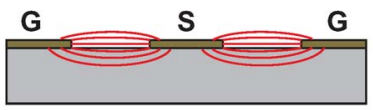

(a)

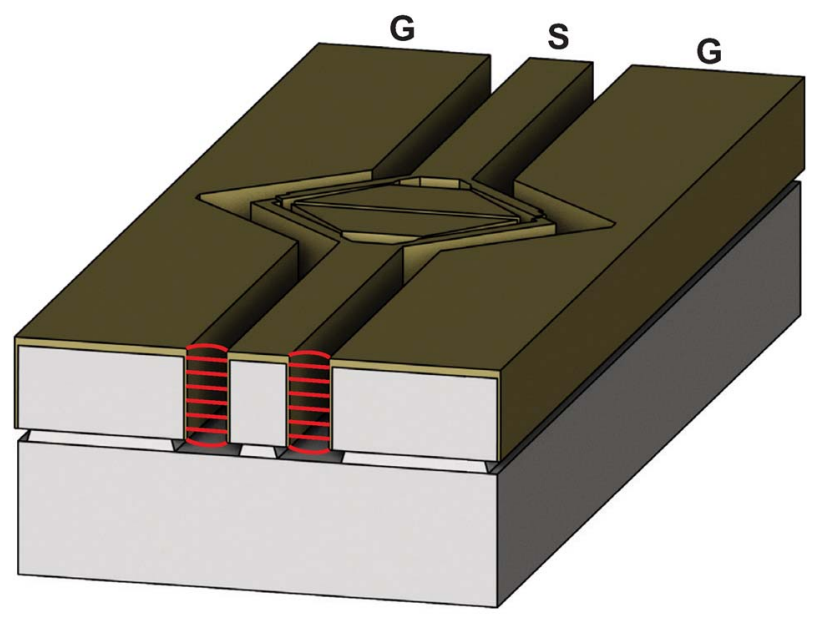

(c)

Fig. 1. Illustration of the difference between 2-D and 3-D transmission lines Transmission losses are lower in the 3-D micromachined SOI RF MEMS transmission line due to much lower electrical field penetration in the substrate and a larger conductor volume for the signal current without skin depth limitations. (a) Two-dimensional transmission line. (b) Three-dimensional transmission line. (c) Axonometric drawing of a 3-D micromachined transmission line with an embedded microdevice.

on the transmission modes and sensitivity of the characteristic impedance of the transmission line, by simulation data and by experimental verification by $\mathrm{RF}$ measurements of fabricated test devices, a comparison of the etch performance to literature data, measurements of the surface profile during etching, and a discussion on yield problems from reduced selectivity due to nonideal isolation of the etched areas.

\section{Metallization Problems in SOI RF MEMS STRUCTURES}

The authors have previously reported on bulk 3-D micromachined CPWs with embedded mechanically bistable RF microelectromechanical systems (MEMS) switches (Fig. 2), based on deep reactive ion etching (DRIE) of a SOI wafer in a single-mask fabrication process shown in Fig. 3 [7], [8].

The patterned 30- $\mu \mathrm{m}$-thick device layer is undercut by wet etching of the SOI buried oxide layer in buffered hydrofluoric acid (BHF) [Fig. 3(c)], which releases moving elements of the switch and also disconnects the metal coating on the device layer sidewalls from the metal layer on the substrate during the subsequent full-wafer sputter metallization [Fig. 3(d)]. While the metal on top and on the sidewalls of the structures in the device layer forms the conductive parts of the transmission line, the metal on the substrate is not wanted. Even though it is disconnected from the metal coatings of the 3-D transmission line, the substrate metal has a negative influence on the transmission line performance. For the ideal transmission line design with no metal on the substrate [Fig. 4(a)], the transmission losses are low since the electric field is mainly confined outside the substrate and the current distribution along the sidewalls is

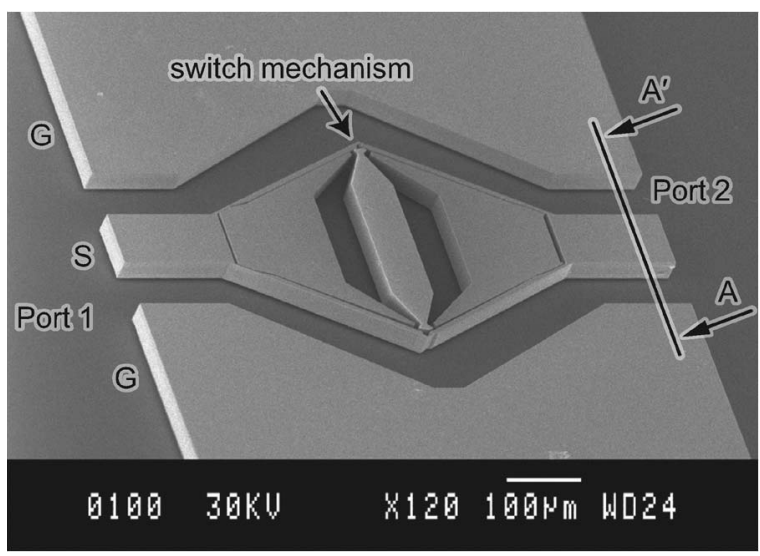

Fig. 2. SEM picture of a 3-D micromachined transmission line with an embedded mechanically bistable RF MEMS switch, fabricated at the Royal Institute of Technology [8].

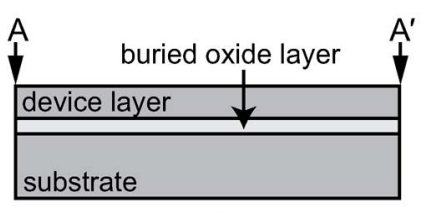

(a)

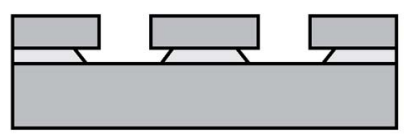

(c)

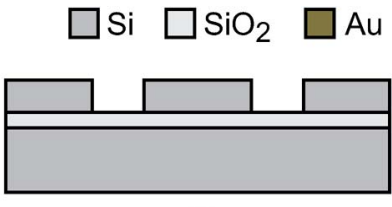

(b)

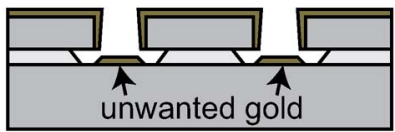

(d)
Fig. 3. Process flow showing the single-mask fabrication of the transmission line on a SOI wafer. The full-wafer metal sputtering step results in unwanted gold on the Si substrate. (a) SOI wafer. (b) DRIE etching. (c) Underetch in BHF. (d) Gold sputtering.

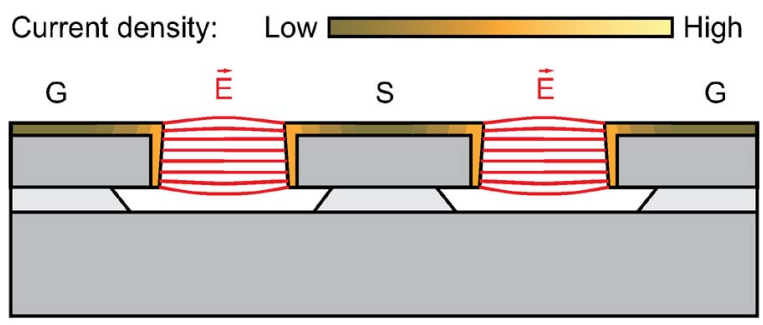

(a)

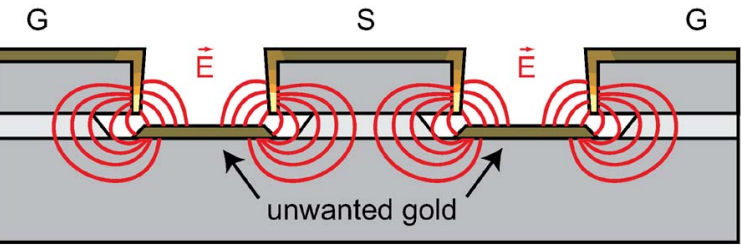

(b)

Fig. 4. Schematic comparison of the electric field lines and current densities in the 3-D CPW, in the cases with and without the gold layer on the substrate. (a) Ideal 3-D CPW design: The electric field lines are almost exclusively confined to the space above the lossy substrate, and the current is distributed uniformly along the sidewalls. (b) Unwanted gold layer on the substrate: Dielectric losses from field lines penetrating the substrate and ohmic losses due to the current crowding on the bottom of the sidewalls.

very uniform. However, in the presence of the unwanted metal areas on the substrate [Fig. 4(b)], there are increased dielectric losses because of the field lines penetrating the silicon substrate, and also, the ohmic losses are increased because of current crowding on the edges of the thin metal layers. 
(a)
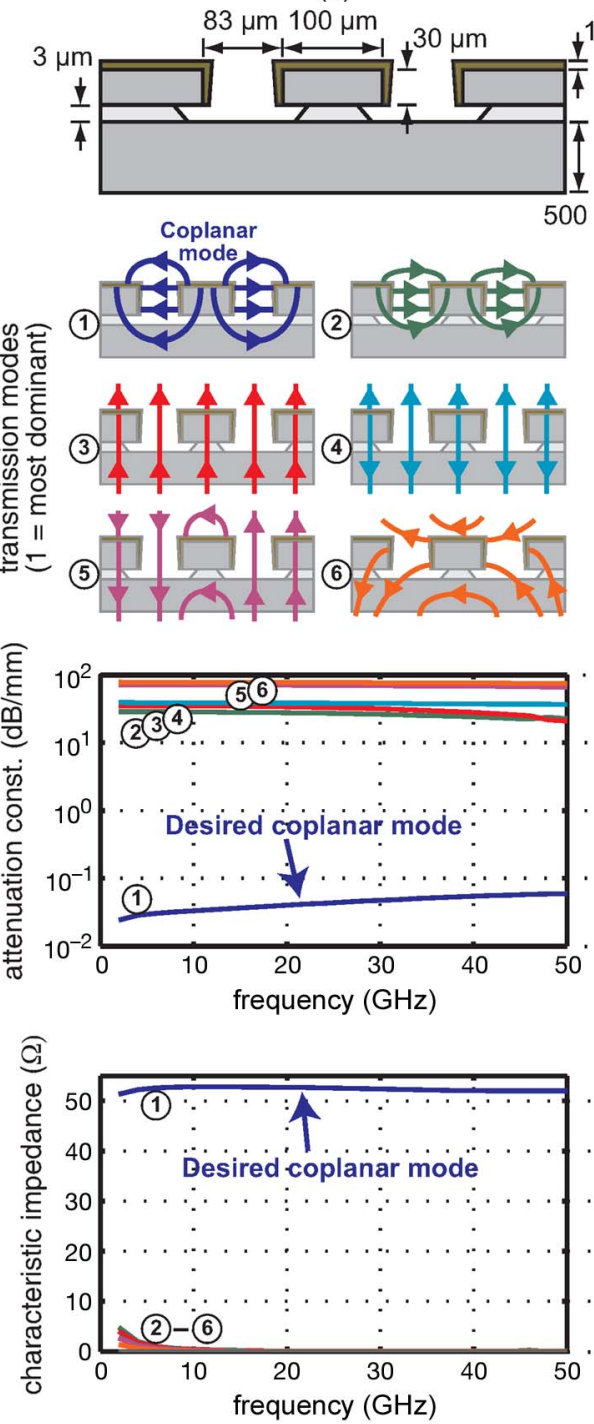

(b)

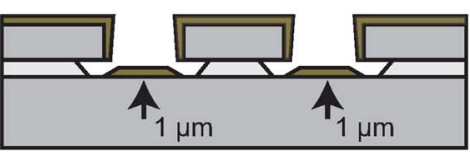

Coplanar
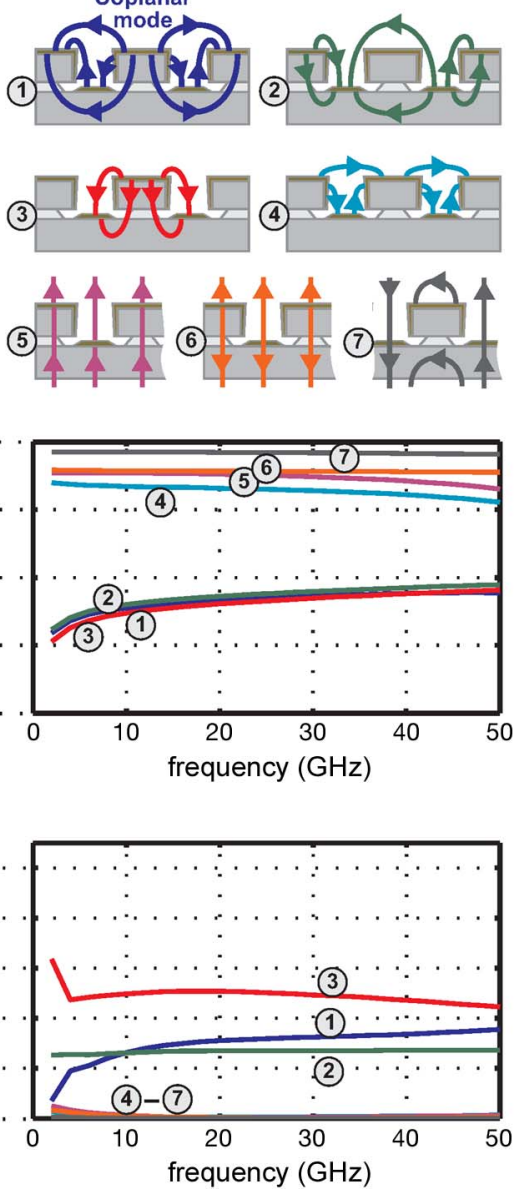

Fig. 5. Comparison of the transmission modes in a CPW with and without residual gold on the handle substrate, extracted from finite-element method (FEM) simulations in ANSYS HFSS. Without the residual gold, the desired coplanar mode is the single dominant mode with lower attenuation of better matching to $50 \Omega$ than all of the modes in the case with the residual gold remaining.

The performance of a waveguide can be judged by its propagation properties, which can be computed from the cross section and the material properties. The ideal waveguide has low attenuation and a constant fabrication-tolerance-insensitive characteristic impedance of typically $50 \Omega$. Furthermore, the waveguide should have a single dominant transmission mode over a large bandwidth to avoid lossy dispersion. The propagation of various $\mathrm{CPW}$ designs has been simulated using ANSYS HFSS. Fig. 5 shows a simulated comparison of the propagation in the CPW with and without the residual gold on the handle substrate. In the case of the residual gold having been removed, the desired CPW mode (1) is clearly dominant, has low attenuation, and is well matched to $50 \Omega$. With the residual gold remaining, there are three dominant modes, which all have much worse attenuation and a characteristic impedance of $13-25 \Omega$ which is difficult to change to $50 \Omega$ for any practically suitable geometry. Fig. 6 shows an attenuation plot for the dominant modes from Fig. 5.

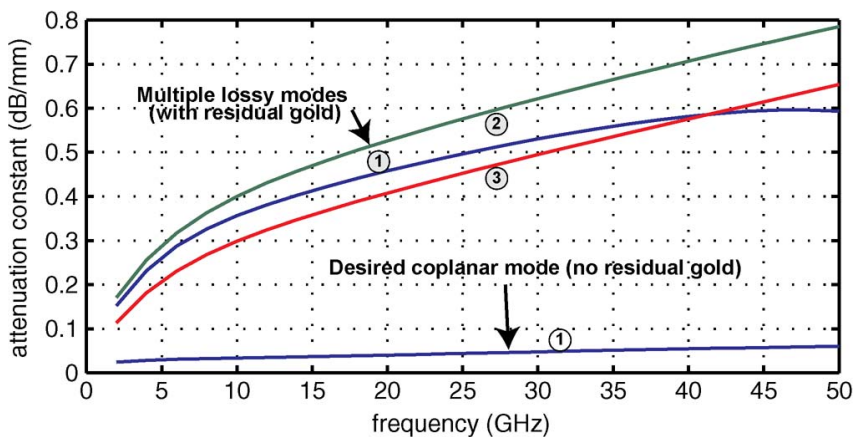

Fig. 6. Comparison of the attenuation of the dominant modes in Fig. 5.

Furthermore, without gold on the substrate, matching the transmission line design to a $50-\Omega$ characteristic impedance is straightforward, for example, by adapting the distance between the signal line and the ground. With the substrate metal layer present, it is almost impossible to adapt the geometry to fit a 


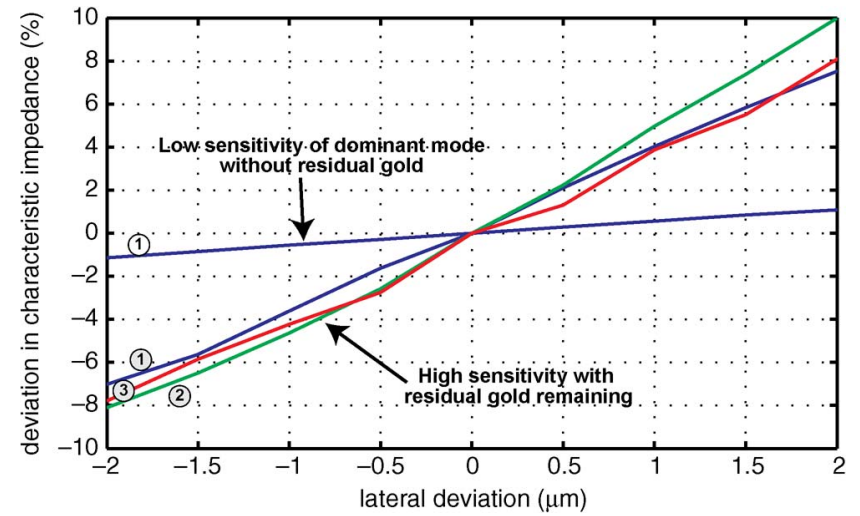

Fig. 7. Sensitivity analysis of the characteristic impedance of the dominant modes with and without residual gold on the handle substrate of the CPW, extracted from a FEM simulation. (Numbers refer to the modes in Fig. 5.)

$50-\Omega$ line because of the too narrow slots. Moreover, since the position of the metal edges is unpredictable, the characteristic impedance is very difficult to control, resulting in increased reflections. Fig. 7 shows the sensitivity of the characteristic impedance to a lateral deviation of the edge of the deposited metal layer. Without the residual gold on the substrate, the sensitivity to fabrication tolerances is eight to ten times lower than that with the residual gold still there, which makes the design more robust.

Unwanted sputtering or evaporation of metal could be avoided by using a shadow mask. However, shadow masking is not suitable for this process since it is not possible to achieve both good step coverage of the sidewalls and avoiding of any deposition on the substrate at the same time. Furthermore, the alignment accuracy needed for this process is not possible with shadow masking.

Photolithography is also unsuitable because of the large step size. Even when using spray coating, it is impossible to protect the sidewalls in narrow high-aspect-ratio trenches without also protecting the metal below the sidewall edges.

Localized electroplating of the gold, instead of full-wafer sputtering, would be possible. However, the roughness of the electroplated gold surface makes it unsuitable for microwave signals, and the seed layer still needs to be removed from the substrate.

As an alternative to SOI wafers, silicon-on-glass substrates could also be used for this RF MEMS device process.

\section{MASKleSS ElectrochemicAlly Assisted Selective Wet Etching}

The etching method for removing unwanted gold areas, as presented in this paper, is based on the electrochemically discriminated dissolution of gold in a chloride solution [16], [20]. Fig. 8 shows the etching setup for etching gold on the substrate of a SOI wafer. A potentiostat circuitry controls the potential between the anode and the saturated calomel reference electrode (SCE) in the $1-\mathrm{mol} \cdot \mathrm{L}^{-1} \mathrm{NaCl}(\mathrm{aq})$ electrolyte. The sample to be etched is submerged into the electrolyte, and the anode is placed in contact with the substrate gold to be etched. Keeping a 1.2-V potential difference to the reference electrode favors the formation of water-soluble $\mathrm{Au}(\mathrm{III})$ complexes at

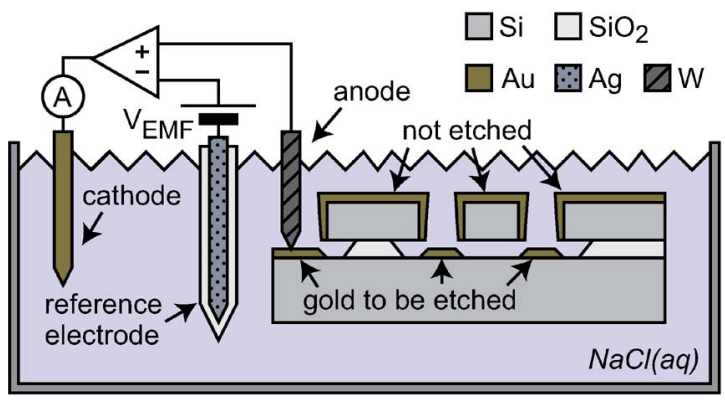

Fig. 8. Electrochemical etching setup used to selectively etch the unwanted gold on the substrate. The gold is dissolved when the anode is kept at $1.2 \mathrm{~V}$ to the reference electrode. Electrically not-connected gold areas are not etched.

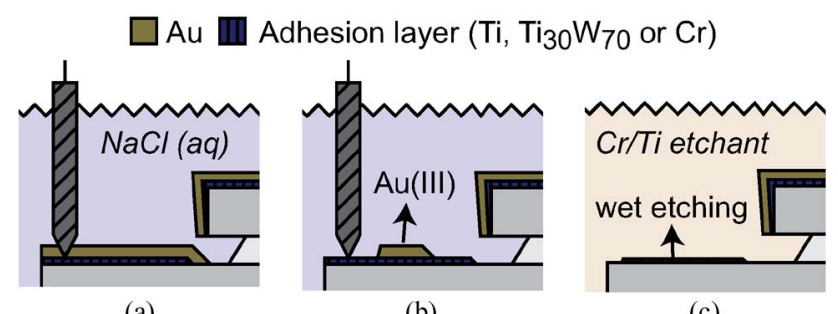

(a)

(b)

(c)

Fig. 9. Since the conductive adhesion layer is not attacked by the electrochemical etching, all gold on the substrate is completely dissolved even if Au islands are formed during the etching. The adhesion layer is later removed using an appropriate wet etchant. (a) $0 \mathrm{~s}$. (b) $\sim 60 \mathrm{~s}$. (c) $\sim 45 \mathrm{~min}$.

the anodic gold-electrolyte interface, dissolving the gold [16]. Only a single anode contact point is necessary to etch all gold areas on the substrate, if these areas are electrically connected in the mask design, which is typical for this type of CPWbased RF MEMS devices. Thus, the electrochemical etching is independent of the substrate conductivity, and a high-resistivity substrate may be chosen for best performance. For designs with closed-loop structures, where different areas on the substrate are isolated by geometry and, thus, electrically from each other, multiple contact points or doped low-resistivity paths in the substrate can be used. The gold areas on the device layer of the SOI wafer are not electrically connected to the anode and, therefore, not attacked. A vacuum chamber treatment of the samples submerged in the $\mathrm{NaCl}(\mathrm{aq})$ solution was necessary to remove air pockets from the narrow waveguide trenches before the etching. A low-pressure level of about 10 mbar was sufficient.

Fig. 9 shows how all gold on the substrate is completely dissolved even if gold islands are formed during the etching. This is guaranteed by the metal adhesion layer for the metallization process, which is not attacked by the electrochemical etching, and thus is electrically connecting even separated gold areas on the substrate. The reasons for the locally faster removal of certain gold areas are nonuniformities in the gold layer thickness and local higher field strengths caused either by surface roughness or by the geometry of the design. The adhesion layer is subsequently removed in a conventional wet etch step, i.e., not electrochemically assisted, as the chemical etchant provides a sufficient etch selectivity between the adhesion layer material and the gold. The adhesion layer below the remaining gold areas is not attacked since it is covered by the gold.

A photograph of the etching setup is shown in Fig. 10. The setup includes a stereomicroscope for inspection during etching, a magnetic stirrer, an elevated positioning platform for 


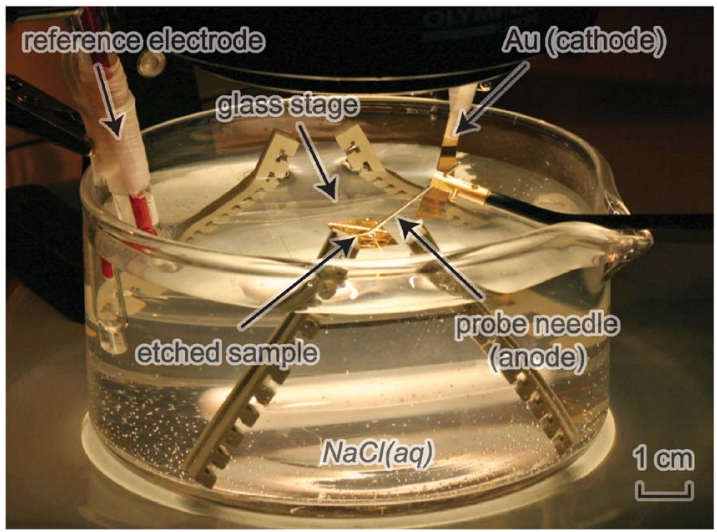

Fig. 10. Photograph of the etching setup. The sample to be etched is placed on a glass stage in the electrolyte. The reference electrode and the Au cathode are inserted into the liquid, and the anodic probe needle is used to contact the specific gold areas to etch.

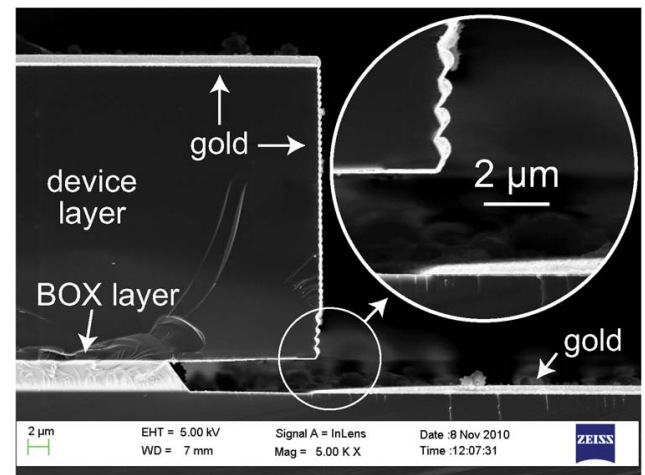

(a)

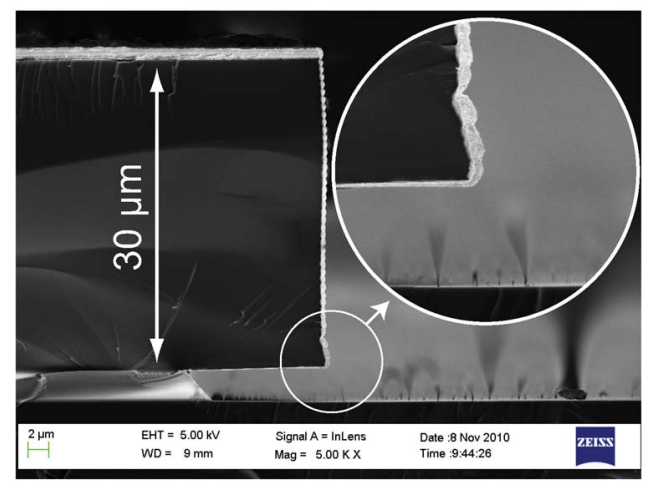

(b)

Fig. 11. SEM pictures of the waveguide before and after the residual gold on the handle layer is removed. (a) Residual gold not removed. (b) Residual gold removed.

probing of the sample in the solution, the reference electrode, and the gold cathode.

SEM pictures of the cross section of a 3-D micromachined waveguide structure before and after the etching are shown in Fig. 11.

\section{RF CHARACTERIZATION}

The need of removing the residual gold from the waveguides was verified by RF characterization of fabricated waveguides, both with and without the residual gold (see Fig. 11), by S-parameter measurements with a two-port network analyzer

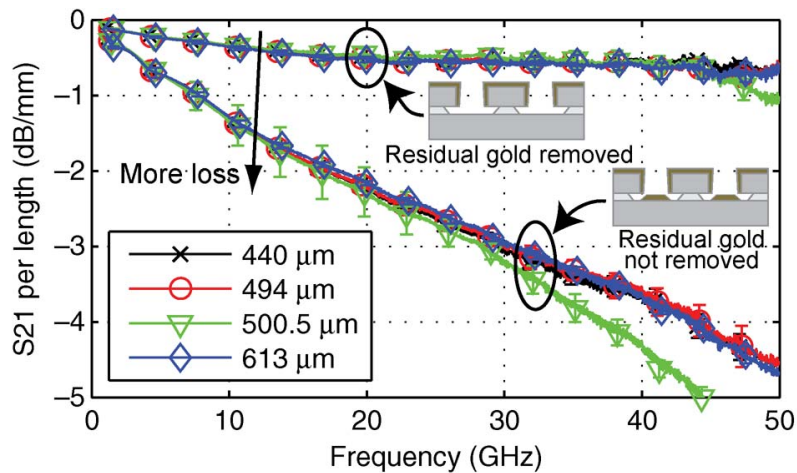

(a)

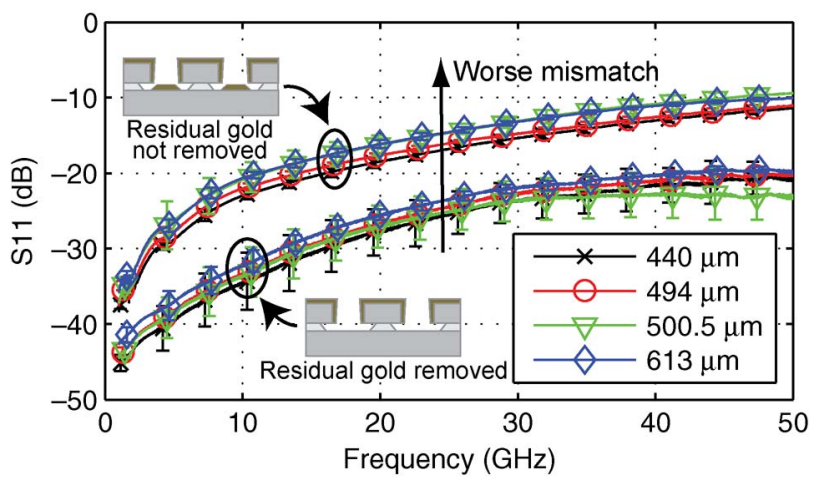

(b)

Fig. 12. RF characterization of 3-D micromachined waveguides with and without removal of the residual gold from the handle layer. Waveguides of four different lengths and multiple waveguides of each length were measured. Averages are shown with $2 \sigma$ error bars. The waveguides without residual gold were significantly less lossy and had less mismatch. (a) Average insertion loss per waveguide length. (b) Average reflections.

and statistical evaluation. The measurement results are shown in Fig. 12. Waveguides of four different lengths were measured, and average transmission parameters for multiple waveguides of each length were obtained and plotted with $2 \sigma$ error bars. Fig. 12(a) shows that the structures with the residual gold not having been removed are clearly more lossy for all samples than the waveguides where the residual gold has been removed. At $10 \mathrm{GHz}$, for instance, the insertion loss is reduced from 1.3 to $0.3 \mathrm{~dB} / \mathrm{mm}$, which corresponds to a reduction in transmission power loss from $25 \%$ to $6.6 \%$ loss per millimeter. Fig. 12(b) shows that the losses really are attributed to radiation and material losses, as the RF reflections, well below $1 \%$ for both cases, are insignificant $(0.6 \%$ for the residual gold not having been removed and $0.05 \%$ for the residual gold having been removed).

Thus, the removal of the residual gold meant a significant improvement in the performance of the transmission line, as predicted by simulations in Section II. The main source of the $0.3-\mathrm{dB} / \mathrm{mm}$ loss of the transmission lines without the residual gold is believed to be that the gold thickness on the sidewalls of the signal line is less than the skin depth, due to unoptimized step coverage in the sputtering process.

\section{Etch Characterization}

To characterize the etching process, three $500-\mu$ m-thick standard silicon wafers were metallized and diced into $1-\mathrm{cm}^{2}$-large test pieces. Three different metallization stacks were applied on 


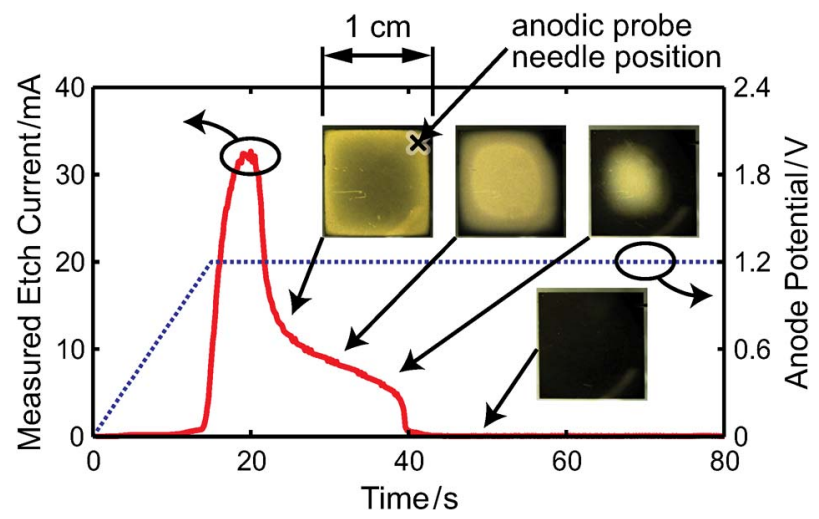

Fig. 13. Measured etch current during etching of a $1-\mathrm{cm}^{2}$ evaporated $\mathrm{Au}$ sample (with a Ti adhesion layer). The inserts show microscope pictures of the sample, taken during the etching.

different test wafers: evaporation of Ti and Au, evaporation of $\mathrm{Cr}$ and $\mathrm{Au}$, and sputtering of $\mathrm{Ti}_{30} \mathrm{~W}_{70}$ and Au. All metal layers were $50 \mathrm{~nm}$ thick, resulting in a total metal thickness of $100 \mathrm{~nm}$.

Fig. 13 shows the etch current measured at the cathode during the etching of the evaporated gold layer on one of the Ti/Au test pieces. The etch rate reaches a maximum of $16 \mathrm{~nm} / \mathrm{s}$ and varies during the process because of the reduction of the etched area. For completely etching a gold layer of a measured thickness of $520 \mathrm{~nm}$, the etch time was 60 s, i.e., the etch rate of the electrochemically assisted etching at a controlled bias potential of $1.2 \mathrm{~V}$ versus $\mathrm{SCE}$ in the $1-\mathrm{mol} \cdot \mathrm{L}^{-1} \mathrm{NaCl}(\mathrm{aq})$ solution was determined by the present work to be $520 \mathrm{~nm} / \mathrm{min}$. The inserts show microscope pictures of the sample, taken during the etching, showing the formation of a gold island because of the gold being etched faster on the edges of the sample. The anodic probe needle is positioned in the top-right corner of the sample, which obviously accelerates the etching in that corner.

Simulation results of a simple numerical etch model, written in MATLAB, are shown in Fig. 14. The model takes into account the $1 / \sqrt{t}$ behavior of saturating diffusion [21] and also approximates the effects of the etch rate being faster at the boundaries of the etched area and additional etch-rate acceleration at the edges of the chip due to increased convection in the agitated solution. The normalized simulated etch current is in good agreement with the measured behavior.

Surface scans of the etched surface, obtained by optical profilometer measurements, are shown in Fig. 15 for different stages of the etching. Also here, it can be seen that the outside of the test chip is etched first and that the gold surface is roughened during the etching.

In Fig. 16, the influence of different adhesion layer materials on the gold etch performance is shown. The gold was etched on three test pieces for each of the three metallization stacks. The graph shows the cumulative etch charge as a function of time, i.e., the time integral of the etch current measured during the etching. The slope of the curves corresponds to the etch speed, and the onset of the final flat of the curves indicates that the etching has stopped. The final level of the curve represents the total amount of transferred charges, which corresponds to some degree to the total amount of removed gold. All of the gold was completely etched on all samples

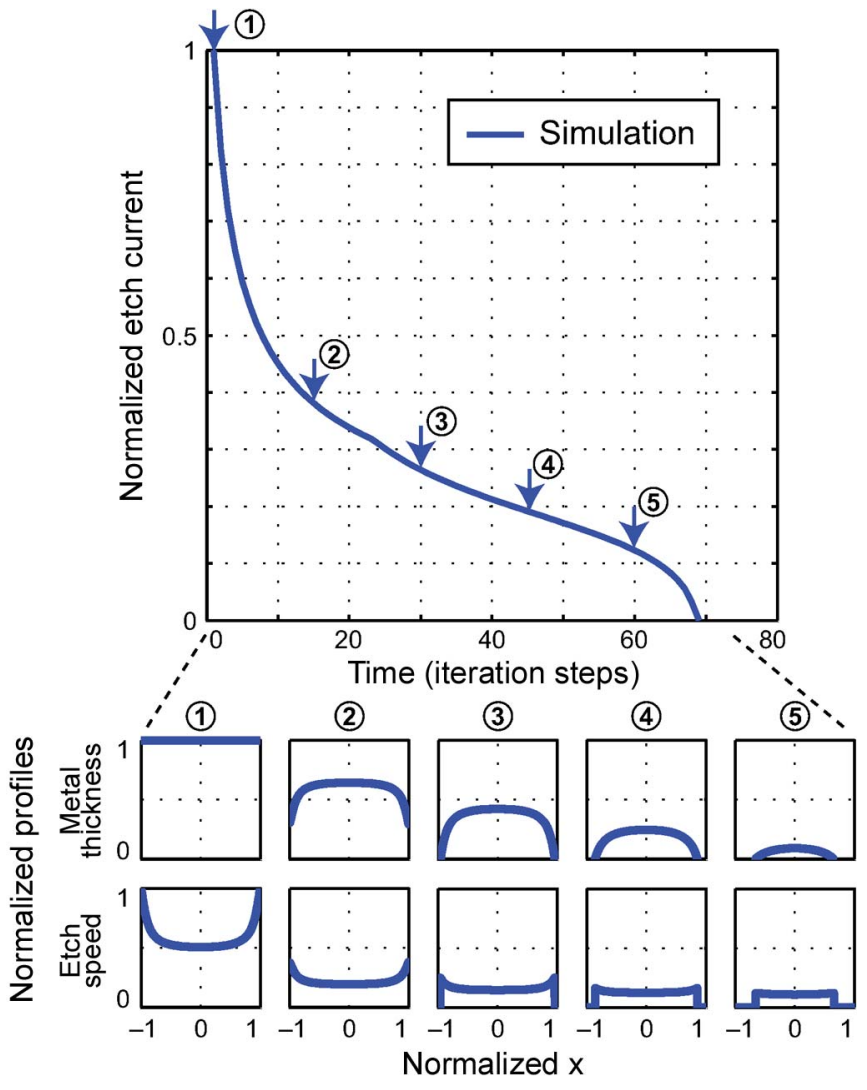

Fig. 14. Simulated etch current based on a simple numerical MATLAB model.

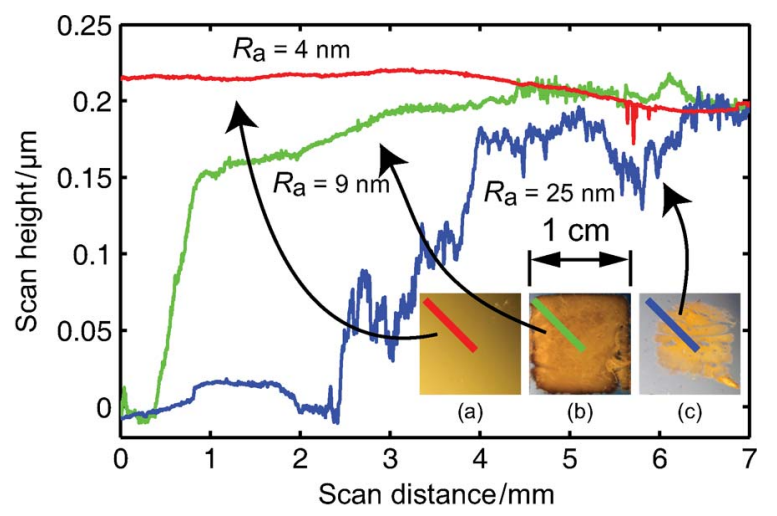

Fig. 15. Optical profilometer surface scans of the etched surface of TiW/Au test pieces that are (a) not etched, (b) only slightly etched, and (c) partially etched through. The local roughness of the surface is shown by the $R_{a}$ values.

based on $\mathrm{Ti}$ and $\mathrm{Ti}_{30} \mathrm{~W}_{70}$ adhesion layers, whereas the gold on two of the Cr-based samples was not etched completely, as indicated by the curves stopping prematurely. Even for the completely etched samples, there is considerable variation in the etch speed and the total etch charge. The performance of the etch is compared to literature values from [16] in Fig. 17 with decent agreement. Fig. 18 shows microscope pictures of the etching on the different adhesion layers.

A possible explanation for the etch speed variation could be a difference in the electrical contact between the anodic probe needle and the metal during different runs. If the contact resistance is high, the etch speed will be decreased because of a 


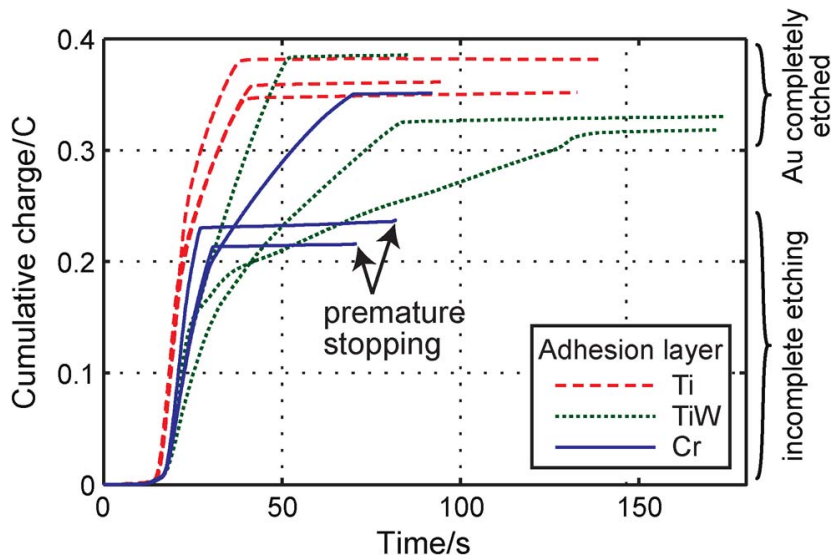

Fig. 16. Comparison of the etch performance of gold on different adhesion layer materials. On all of the samples using Ti and TiW, the gold was etched completely, whereas on most of the $\mathrm{Cr}$ samples, the gold etching stopped prematurely.

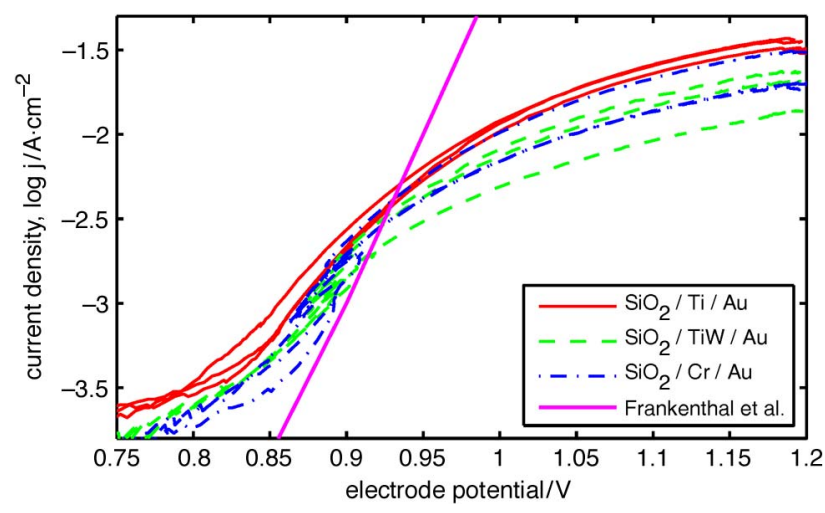

Fig. 17. Current density as a function of electrode potential for the different test samples, compared to the data from [16].

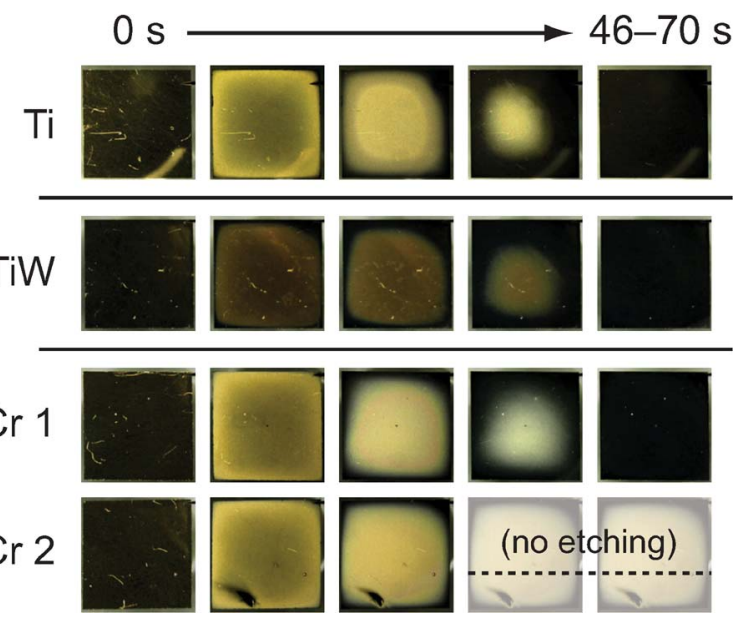

Fig. 18. Microscope pictures taken during the etching of the gold on different adhesion layer materials.

larger voltage drop. A source of error in measuring the total etch charge could be the additional electrolysis of $\mathrm{H}_{2} \mathrm{O}$ into $\mathrm{H}_{2}$ and $\mathrm{O}_{2}$, which theoretically occurs already at $1-\mathrm{V}$ potential difference to the reference electrode [22]. The premature stopping of the etching on the samples with a $\mathrm{Cr}$ adhesion layer is explained by the electrochemical etching also attacking the

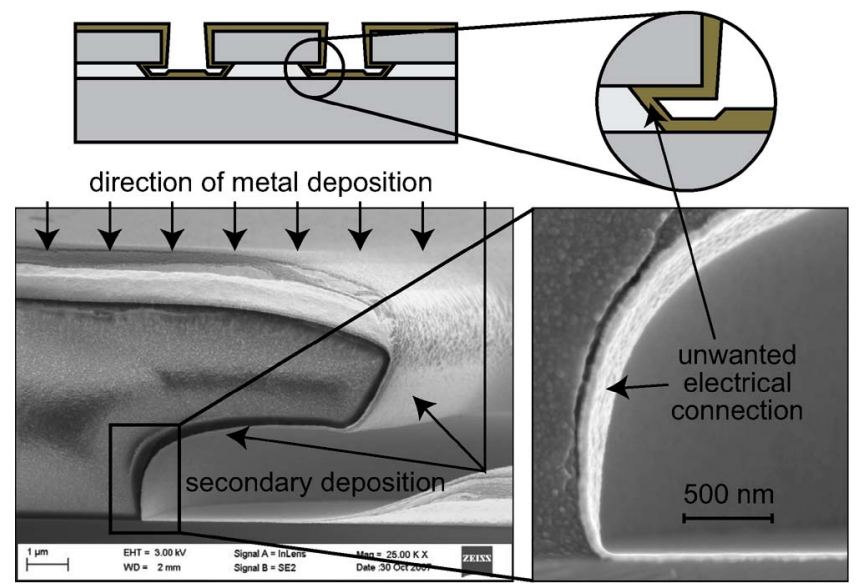

Fig. 19. SEM picture of an overhang test structure metallized by sputter deposition of gold. Even though the metal is deposited from the top, there is a continuous layer deposited even in the inner part of the shadowed area. Thus, under these sputtering conditions, there will be a thin electrical bridge reducing the selectivity between the gold areas when etched electrochemically.

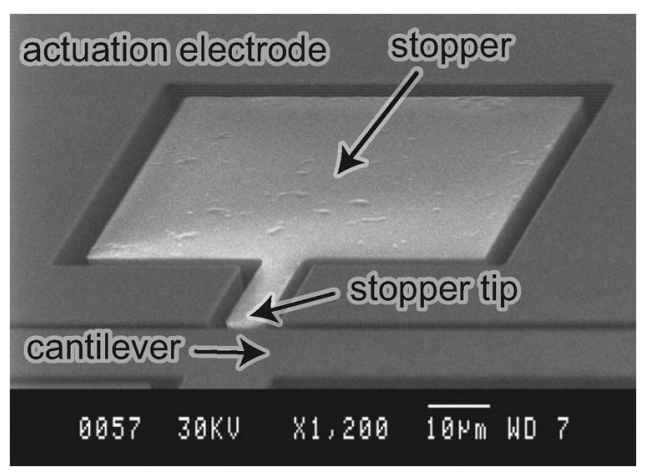

Fig. 20. SEM picture of a stopper used to prevent short circuit between the switch cantilever and the actuator electrode.

adhesion layer: Literature values for the anodic dissolution of chromium in chloride solutions show that it is attacked at a rate of $10^{-2} \mathrm{~A} / \mathrm{cm}^{2}$ which is comparable to that of gold at $1.2 \mathrm{~V}$ [16], [23], and thus, parts of the sample become completely isolated from the anode and are not further etched. Ti and TiW have a much higher resistance to chloride corrosion: The current densities at $1.2 \mathrm{~V}$ are approximately $10^{-5} \mathrm{~A} / \mathrm{cm}^{2}$ [24] and $10^{-4} \mathrm{~A} / \mathrm{cm}^{2}$ [25], respectively, i.e., the selectivities of these layers to gold are about $1: 1000$ and $1: 100$, respectively.

The etch selectivity to gold areas on the device layer which are not electrically connected to the anode was determined by measuring line scans of the surface profile of biased and unbiased samples, before and after etching, using an optical profilometer. No detectable etching of the unbiased sample could be seen even after 1-h etching time (the total etching after $1 \mathrm{~h}$ was smaller than the surface roughness of less than $3 \mathrm{~nm}$ ). Furthermore, no detectable etching of unbiased gold areas in close proximity to the biased areas, which were etched at $520 \mathrm{~nm} / \mathrm{min}$, was seen on test samples featuring a $500-\mathrm{nm}$ gold layer on a $1-\mu \mathrm{m}$ oxidized low-resistivity silicon substrate, in which $40-\mu \mathrm{m}$-wide and $125-\mu \mathrm{m}$-deep dicing lines were cut to isolate the neighboring areas for the purpose of measuring the etching selectivity. 

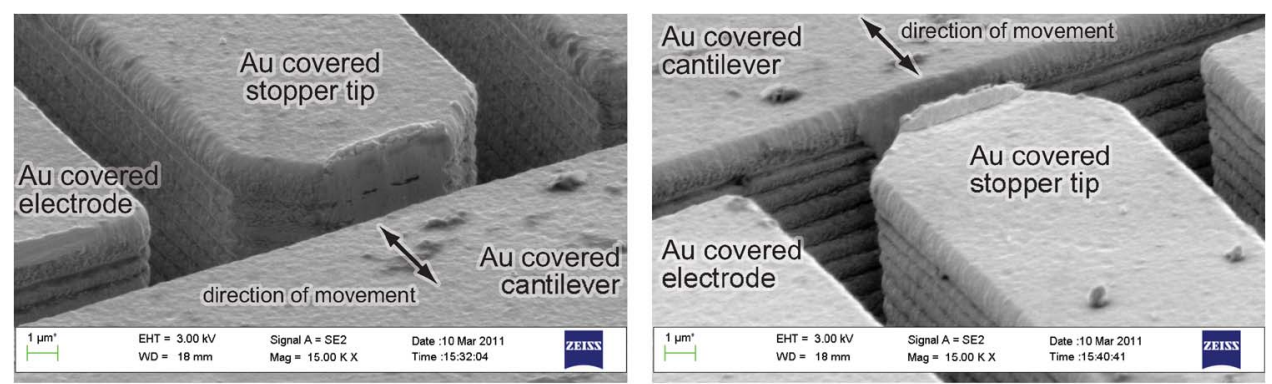

(a)
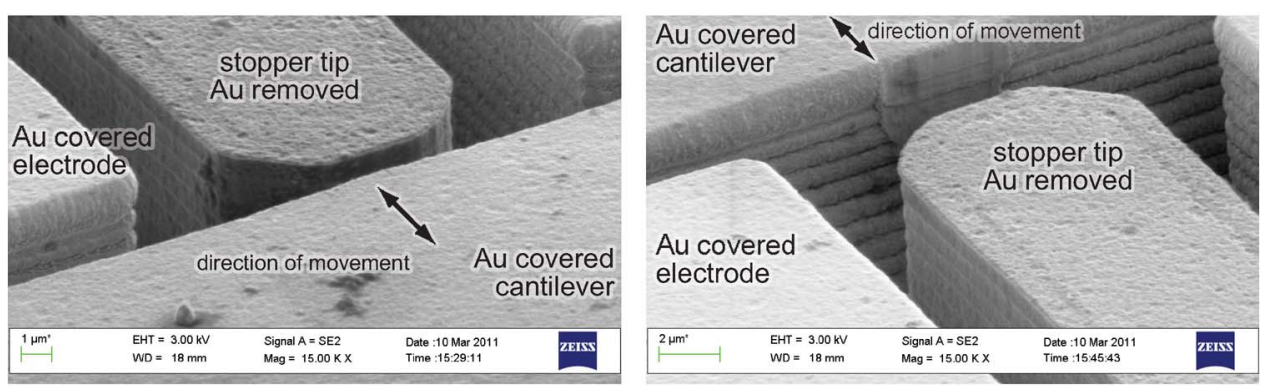

(b)

Fig. 21. Selective removal of the gold coating from the stopper drastically reduces the stopper wear observed after 150 million switching cycles. The stopper tip with gold shows heavy contact wear, whereas there are no signs of wear on the stopper tip where the gold coating has been removed. The deformation of the gold on the sidewall of the moving cantilever, where the gold is not removed, can be clearly seen in both cases. (a) Gold-coated stopper and cantilever, after 150 million actuation cycles. (b) Stopper with gold locally removed, after 150 million actuation cycles.

\section{Vi. Considerations on the Step Coverage OF THE METALLization LAYERS}

The SOI RF MEMS 3-D transmission line structure (see Fig. 1) requires a certain step coverage in the metallization of the device layer in order to sufficiently cover the sidewalls of the transmission line. However, if the step coverage is too high, a thin metallic interconnection between the device layer and the handle layer of the structures might short-circuit these metal layers, which means that gold on the device layer would also be etched when the gold on the handle layer is electrochemically etched. This issue was observed on some of the chips where, while most isolated elements remained unetched, some waveguide elements were visibly attacked and some were completely etched. Fig. 19 shows a SEM picture of a photoresist test structure with a 5- $\mu \mathrm{m}$ overhang, where even the most shadowed part is coated due to resputtering from the handle substrate during the metal coating. Appropriate tuning of the deposition parameters avoids this problem, resulting in electrically isolated areas as shown in Fig. 11.

\section{LOCAL REMOVAL OF GOLD ON INDIVIDUAL DEVICE LAYER ELEMENTS}

In addition to the removal of the gold on the handle layer of SOI RF MEMS devices, as demonstrated in the previous sections of this paper, the method can also be used to locally remove gold from individual isolated elements in the device layer. This is shown in this section for removing gold on individual stoppers (Fig. 20) of electrostatic actuators without isolation layers, reducing the wear between these nonelectrically functioning mechanical contact points [8]. Repeated actuation causes mechanical wear of the gold on the stopper tips which increases the susceptibility of stiction and thus drastically reduces lifetime.

The electrochemically assisted etching is applied to locally removing the gold on the stoppers. For the etching, the top gold surface of the stoppers to be etched was directly contacted with the anodic probe needle. To judge the effect on the mechanical wear of the stopper tips, test devices with or without gold on their stoppers were actuated for 150 million cycles at $3-\mathrm{kHz}$ switching frequency. Even though stiction was not observed for any device for these low cycle numbers, a clear difference in the stopper tip wear was observed. Fig. 21(a) shows heavy contact wear of a gold-covered stopper tip, whereas Fig. 21(b) shows no signs of wear after 150 million cycles for a stopper tip where the gold coating had been removed. A material analysis was performed using an Oxford INCA EDX system, showing that the gold had been removed from the stopper and its sidewalls. A comparison between unactuated and actuated stopper tips indicated that some gold is transferred from the still gold-coated cantilever side to the stopper tip during the 150 million cycles.

\section{CONCLUSION}

A novel method for selective local removal of metal layers using maskless electrochemically assisted wet etching has been presented and successfully applied to the fabrication of 3-D micromachined SOI RF MEMS transmission lines with embedded laterally actuated microswitches. RF characterization of the transmission lines shows good correspondence with simulations and verifies the need for etching the metal layers. Furthermore, selective removal of the metallization on stoppers has been shown to drastically reduce the mechanical wear on the stopper tip. 


\section{REFERENCES}

[1] M. Sterner, N. Roxhed, G. Stemme, and J. Oberhammer, "Maskless selective electrochemically assisted wet etching of metal layers for 3D micromachined SOI RF MEMS devices," in Proc. IEEE Int. Conf. MEMS, Tucson, AZ, Jan. 2008, pp. 383-386.

[2] C. P. Wen, "Coplanar waveguide: A surface strip transmission line suitable for nonreciprocal gyromagnetic device applications," IEEE Trans. Microw. Theory Tech., vol. MMS-17, no. 12, pp. 1087-1090, Dec. 1969.

[3] I. Llamas-Garro and A. Corona-Chavez, "Micromachined transmission lines for millimeter-wave applications," in Proc. Int. Conf. CONIELECOMP, Puebla, Mexico, Feb. 2006, p. 15.

[4] Y. Zhao, Y.-K. Yoon, X. Wu, and M. G. Allen, "Metal-transfermicromolding of air-lifted RF components," in Proc. TRANSDUCERS, Lyon, France, Jun. 2007, pp. 659-662.

[5] K. Herrick, T. Schwarz, and L. Katehi, "Si-micromachined coplanar waveguides for use in high-frequency circuits," IEEE Trans. Microw. Theory Tech., vol. 46, no. 6, pp. 762-768, Jun. 1998.

[6] A. Liu, M. Tang, A. Agarwal, and A. Alphones, "Low-loss lateral micromachined switches for high frequency applications," J. Micromech. Microeng., vol. 15, no. 1, pp. 157-167, Jan. 2005.

[7] J. Oberhammer, M. Tang, A.-Q. Liu, and G. Stemme, "Mechanically tristable, true single-pole-double-throw (SPDT) switches," J. Micromech. Microeng., vol. 16, no. 11, pp. 2251-2258, Nov. 2006.

[8] M. Sterner, N. Roxhed, G. Stemme, and J. Oberhammer, "Static zeropower-consumption coplanar waveguide embedded DC-to-RF metalcontact MEMS switches in two-port and three-port configuration," IEEE Trans. Electron Devices, vol. 57, no. 7, pp. 1659-1669, Jul. 2010.

[9] M. Datta and D. Landolt, "Fundamental aspects and applications of electrochemical microfabrication," Electrochim. Acta, vol. 45, no. 15/16, pp. 2535-2558, Jul. 2000.

[10] D. Landolt, "Electrodeposition science and technology in the last quarter of the twentieth century," J. Electrochem. Soc., vol. 149, no. 3, pp. S9S20, Apr. 2002

[11] L. T. Romankiw, "A path: From electroplating through lithographic masks in electronics to LIGA in MEMS," Electrochim. Acta, vol. 42, no. 20-22, pp. 2985-3005, 1997.

[12] A. Rogner, J. Eicher, D. Munchmeyer, R. P. Peters, and J. Mohr, "The LIGA technique-What are the new opportunities," J. Micromech. Microeng., vol. 2, no. 3, pp. 133-140, Sep. 1992.

[13] W. J. McTegart, The Electrolytic and Chemical Polishing of Metals. London, U.K.: Pergamon, 1956.

[14] R. Schuster, V. Kirchner, P. Allongue, and G. Ertl, "Electrochemical micromachining," Science, vol. 289, no. 5476, pp. 98-101, Jul. 2000.

[15] A. C. West, C. Madore, M. Matlosz, and D. Landolt, "Shape changes during through-mask electrochemical micromachining of thin metal films," J. Electrochem. Soc., vol. 139, no. 2, pp. 499-506, Feb. 1992.

[16] R. P. Frankenthal and D. J. Siconolfi, "The anodic corrosion of gold in concentrated chloride solutions," J. Electrochem. Soc., vol. 129, no. 6, pp. 1192-1196, Jun. 1982.

[17] S. Ye, C. Ishibashi, K. Shimazu, and K. Uosaki, "An in situ electrochemical quartz crystal microbalance study of the dissolution process of a gold electrode in perchloric acid solution containing chloride ion," J. Electrochem. Soc., vol. 145, no. 5, pp. 1614-1623, May 1998.

[18] Z. Hu and T. Ritzdorf, "Cyanide- and thiourea-free electrochemical etching of gold for microelectronics applications," J. Electrochem. Soc., vol. 154, no. 10, pp. D543-D549, Aug. 2007.

[19] H. Gradin, S. Braun, G. Stemme, and W. van der Wijngaart, "Localized removal of the $\mathrm{Au}-\mathrm{Si}$ eutectic bonding layer for the selective release of microstructures," J. Micromech. Microeng., vol. 19, no. 10, p. 105014 , Oct. 2009.

[20] J. T. Santini, Jr., A. C. Richards, R. Scheidt, M. J. Cima, and R. Langer, "Microchips as controlled drug-delivery devices," Angew. Chem. Int. Ed., vol. 39, no. 14, pp. 2396-2407, Jul. 2000.

[21] C. H. Hamann, A. Hamnett, and W. Vielstich, Electrochemistry, 2nd ed. Weinheim, Germany: Wiley-VCH, 2007.

[22] S. Licht, L. Halperin, M. Kalina, M. Zidman, and N. Halperin, "Electrochemical potential tuned solar water splitting," Chem. Commun., no. 24, pp. 3006-3007, Nov. 2003.

[23] N. D. Greene, C. R. Bishop, and M. Stern, "Corrosion and electrochemical behavior of chromium-noble metal alloys," J. Electrochem. Soc., vol. 108, no. 9, pp. 836-841, Sep. 1961.

[24] S. Y. Yu and J. R. Scully, "Corrosion and passivity of Ti-13in comparison to other biomedical implant alloys," Corrosion, vol. 53, no. 12, pp. 965976, Dec. 1997.

[25] J. Bhattarai, E. Akiyama, A. Kawashima, K. Asami, and K. Hashimoto, "The corrosion behavior of sputter-deposited amorphous W-Ti alloys in $6 \mathrm{M} \mathrm{HCl}$ solution," Corros. Sci., vol. 37, no. 12, pp. 2071-2086, Dec. 1995.

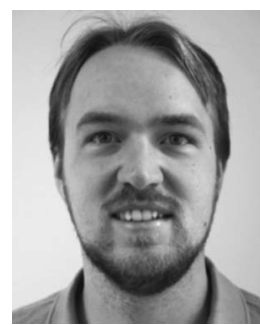

Mikael Sterner was born in Stockholm, Sweden, in 1981. He received the M.Sc. degree in engineering physics from the Royal Institute of Technology (KTH), Stockholm, in 2006, where he has been working toward the Ph.D. degree in microsystems technology in the Microsystem Technology Laboratory since 2006 .

His main research fields include radio-frequency microelectromechanical systems (MEMS) switches and microwave MEMS tunable high-impedance surfaces.

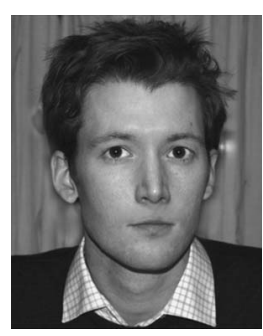

Niclas Roxhed (M'09) was born in Stockholm, Sweden, in 1978. He received the M.Sc. degree in electrical engineering and the Ph.D. degree in microsystem technology from the Royal Institute of Technology (KTH), Stockholm, in 2003 and 2007, respectively.

$\mathrm{He}$ is currently an Assistant Professor at KTH, where he is leading research activities in medicalrelated microelectromechanical systems (MEMS). His main research fields include sensors for medical diagnostics and medical-aid microsystems. He is also involved in high-precision etching using deep reactive ion etching for radiofrequency MEMS switches and 3-D integration of MEMS on ICs for infrared imagers. He has authored or coauthored 39 scientific papers.

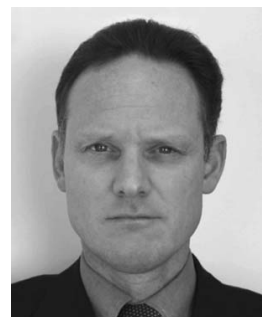

Göran Stemme (M'98-SM'04-F'06) received the M.Sc. degree in electrical engineering and the Ph.D. degree in solid-state electronics from Chalmers University of Technology, Gothenburg, Sweden, in 1981 and 1987, respectively.

In 1981, he was with the Department of Solid State Electronics, Chalmers University of Technology, where he was an Associate Professor (docent) heading the silicon sensor research group in 1990. Since 1991, he has been a Professor at the Royal Institute of Technology (KTH), Stockholm, Sweden, where he is the Head of the Microsystem Technology Lab in the School of Electrical Engineering. His research is devoted to microsystem technology based on micromachining of silicon. The work spans a broad range of technological and application fields, such as medical technology, biochemistry, biotechnology, microfluidics, optical applications, wafer-level packaging, and device integration. Some of the results have successfully been commercialized. $\mathrm{He}$ has published more than 260 research journal and conference papers and is the holder of more than 22 patent proposals or granted patents. He was a member of the Editorial Board of the Royal Society of Chemistry journal Lab on a Chip between 2000 and 2005.

Dr. Stemme is a member of the Royal Swedish Academy of Sciences (KVA) Between 1995 and 2001, he was a member of the International Steering Committee of the IEEE Micro Electro Mechanical Systems Conference series, and he was the General Cochair of that conference in 1998. He has been a member of the Editorial Board of the Journal of Microelectromechanical SYSTEMS since 1997. In 2001, he won, together with two colleagues, the final of the Innovation Cup in Sweden. 


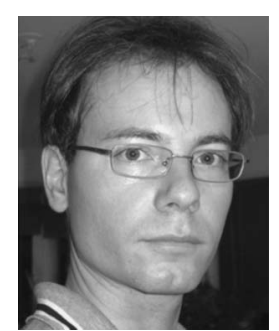

Joachim Oberhammer (M'06) was born in Italy in 1976. He received the M.Sc. degree in electrical engineering from Graz University of Technology, Graz, Austria, in 2000, and the Ph.D. degree from the Royal Institute of Technology (KTH), Stockholm, Sweden, in 2004, for his work on radio-frequency (RF) microelectromechanical systems (MEMS) switches and microsystem packaging.

He was working with automotive sensor electronics and RF identification systems at Graz University of Technology and Vienna University of Technology, Vienna, Austria, before he joined the Microsystem Technology Laboratory, KTH. He was a Postdoctoral Research Fellow at Nanyang Technological University, Singapore. Since 2005, he has been with KTH, where he was an Assistant Professor, has been an Associate Professor since 2007, and is heading a research team with activities in RF and microwave MEMS. In 2007, he was a Research Consultant at Nanyang Technological University. In 2008, he was a Guest Researcher at Kyoto University, Kyoto, Japan, where he spent seven months. He is the author or coauthor of more than 60 reviewed research papers and is the holder of four patents.

Dr. Oberhammer has been a Steering Group member of the IEEE Microwave Theory and Techniques Society (MTT-S) and IEEE Antennas and Propagation Society (AP-S) Chapters in Sweden since 2009. He was a recipient of the Ericsson Research Foundation Award in 2004, a grant by the Swedish Innovation Bridge in 2007, and a visiting researcher scholarship from the Japanese Society for the Promotion of Science in 2008. The research work he is heading received the Best Paper Award at the IEEE European Microwave Integrated Circuit Conference in 2009, the Best Student Paper Award at the IEEE AsiaPacific Microwave Conference in 2010, and Graduate Fellowships from the IEEE MTT-S in 2010 and in 2011. He served as a TPRC member of IEEE Transducers 2009, IEEE International Microwave Symposium 2010 and 2011, and IEEE Micro Electro Mechanical Systems 2011. 